\title{
Review of Sleep Problems Related to Social Distancing and Home Confinement During the COVID-19 Pandemic
}

\author{
Wan Seok Seo \\ Department of Psychiatry, Yeungnam University College of Medicine, Daegu, Korea
}

\begin{abstract}
Since the first outbreak of coronavirus disease (COVID-19) in December 2019, we have experienced many changes that we have never experienced before. Governments around the world have instituted various forms of social isolation measures to prevent the spread of COVID-19. These measures include social distancing, home confinement, quarantine, and lockdown. Unfortunately, these measures have increased the ambiguity of the economic situation and the uncertainty regarding the future. For many individuals, these actions resulted in changes in routines at home, school, and work; changes in roles at home, mental health, and physical activity; and changes in circadian cues. All of these alterations resulted in disruptions in sleep patterns and sleep difficulties. In this paper, the alterations in sleep patterns and the sleep difficulties related to social isolation policies would be reviewed. These include social distancing and home confinement. Finally, methods to improve sleep problems also reviewed.
\end{abstract}

Keywords: COVID-19; Social distancing; Home confinement; Sleep; Mental health

Received: August 31, 2021 Revised: September 9, 2021 Accepted: September 9, 2021

Corresponding author: Wan Seok Seo, MD, PhD, Department of Psychiatry, Yeungnam University College of Medicine, 170 Hyeonchung-ro, Nam-gu, Daegu 42415, Korea.

Tel: 82-53-620-3340, Fax: 82-53-657-3921, E-mail: sws3901@ynu.ac.kr

@ This is an Open Access article distributed under the terms of the Creative Commons Attribution Non-Commercial License (https://creativecommons.org/licenses/by$\mathrm{nc} / 4.0)$ which permits unrestricted non-commercial use, distribution, and reproduction in any medium, provided the original work is properly cited.

\section{INTRODUCTION}

In December 2019, coronavirus disease (COVID-19) was first reported in Wuhan, China. COVID-19 is highly contagious and spread rapidly around the world in a brief amount of time [1]. The World Health Organization (WHO) declared COVID-19 a global pandemic on March 11, 2020. The WHO recommended various social isolation measures such as social distancing, self-isolation, and quarantine of those who had contracted or potentially contracted COVID-19. These measures were put in place to prevent the spread of the virus. During the COVID-19 pandemic, following personal hygiene guidelines, social distancing, wearing masks, remaining in quarantine in case of infection, and switching in-person school classes to online classes are all crucial measures to prevent the spread of infection [2]. Many measures instituted for the prevention of COVID-19 epidemics have resulted in fundamental changes to individual lifestyles, educational systems, public health, and the economic environment. Many people live in a society with increased uncertainty and experience concerns regarding COVID-19 infection. They suffer from fatigue related to the long-term social controls. Preventive measures such as social distancing and home confinement play a beneficial role in preventing the spread of COVID-19. However, these measures have had a negative impact. These actions interfere with individuals' outside activities and reduce their daylight exposure [3,4].

Sleep is an essential physiological phenomenon for the proper functioning of humans. Circadian rhythm is a mechanism that allows people to sleep and wake regularly. It is primarily affected by daylight; however, it is also impacted by mealtimes, exercise, and social activities [5,6]. Home confinement can negatively affect an individual's circadian rhythm homeostasis, psychological functioning, mental health, as well as decision making [6,7].

In this paper, the effects of social distancing and home confinement, which are measures to prevent the spread of COVID-19, on individuals' lifestyle, mental health, and sleep will be reviewed. Methods to overcome sleep problems will also be reviewed. 


\section{LIFESTYLE CHANGE AND STRESS DUE TO SOCIAL DISTANCING AND HOME CONFINEMENT}

To prevent the spread of COVID-19, many countries have implemented social distancing and home confinement for work and school for an unprecedented amount of time. As these measures are prolonged, individuals' stress increases. People go out less often and spend an increasing amount of time at home. Students take classes online or are home schooled instead of attending classes. Working parents are increasingly working from home. Family members share the limited space of home for extended periods. They endure large amounts of time at home without being exposed to new stimuli. Family members experience disruption in their structured routines. This is stressful for the entire family. While confined at home, students cannot play, go out, or engage in sports activities with their friends. Parents must balance work and home responsibilities, such as work assignments, childcare, and housework while working from home. This reduces work productivity and efficiency and increases conflict within the family. Parenting responsibilities are most often shouldered by women who have children [5].

Changes in the economic situation are also stressful for many people [8]. In the United States, job losses due to the COVID-19 pandemic are the greatest since the Great Depression. The prolonged stay-at-home/lockdown caused by the COVID-19 pandemic has also had a devastating effect on the world economy. The self-employed, start-ups, entertainment companies, hospitality establishments, bars, restaurants, and other enterprises that were forced to close are experiencing particularly severe economic hardship. Many of their employees are at risk of job insecurity and unemployment [5].

Socially, COVID-19 has brought about lifestyle changes such as cessation of social interaction and formation of positive relationships, severance of creativity and opportunities, and restrictions on physical activity (PA) and mobility [6]. Social distancing and home confinement have limited opportunities to interact with friends and relatives. It has also restricted participation in leisure activities such as shopping, attending cultural activities, eating out, and watching sports [5]. In Korea, daily living, leisure, social, and education activity of adults with COVID-19 has decreased [9].

Eating, exercising, and leisure activities are more pleasant when enjoyed with friends. Social distancing negatively affects an individual's emotions by limiting pleasurable social activities [10]. Additionally, people consume negative news related to COVID-19 through TV, electronic media, and social networking services for extended periods of time. This promotes negative emotions and cognitive narrowing.

Increased stress negatively affects an individual's health. An increase in stress increases the likelihood of a decrease in PAs, an increase in health risks, an increase in alcohol consumption, and an increase in domestic violence. These factors have a bidirectional effect on sleep disturbances [11-13]. Also, stress itself negatively affects sleep quality, timing, and duration [14].

CHANGES IN SLEEP PATTERNS
AND SLEEP PROBLEMS DUE TO
SOCIAL DISTANCING AND HOME
CONFINEMENT

During the COVID-19 pandemic, many people have experienced difficulty sleeping and having adequate sleep time [5]. In a survey of 1,024 adults over the age of 18 using the Survey Monkey platform, $23.4 \%$ of participants reported that their sleep quality deteriorated after the COVID-19 pandemic [15]. In a survey of 1,013 English-speaking US adults, 56\% of participants currently experience some evidence of insomnia. They are more worried than they were prior to COVID-19. The greater their insomnia, their suicidal thoughts were significantly higher [16]. In a study of the Greek population, $38 \%$ of subjects reported clinical insomnia. This insomnia was associated with loneliness, uncertainty, and depression [17]. In a systematic review of 54,231 subjects in 44 papers, the pooled prevalence rate of sleep problems was $35.7 \%$ in all populations. The pooled prevalence rate of sleep problems in the general population was $32.3 \%$ and $36.0 \%$ among healthcare workers. However, it was very high in patients with COVID-19 (74.8\%). The prevalence of sleep problems differed depending on the evaluation tool. The Pisstburgh Sleep Quality Index-based measurements (39.6\%) were higher than researcher-developed measurements (25.2\%) [18].

Sleep problems during home confinement had a greater impact on those who had difficulty accessing the healthcare system or those at higher risk [19]. In general, women had more insomnia and poorer sleep quality than men. In particular, sleep disruption and insomnia were more frequent in pregnant women or in mothers less than 1 year after birth [20,21]. People who lived in poor home environments such as those with small windows or had poor visibility to the outside also had more sleep problems [5].

There are several reasons for this. First, sleep problems are related to changes in PA. Low levels of activity due to depression or confinement, as well as too high a level of activity due to work overload all negatively affect sleep. However, daytime PA improves sleep quality [22]. In a study on the association between PA and mental health/sleep with 1,281 adults in Norway, physically active adults had lower anxiety and depression symptoms. Anxiety and depressive symptoms were associated with sleep disturbances [23]. Increased PA and outdoor activity increase melatonin release, which plays a key role in inducing sleepiness. On the other hand, a decrease in PA may increase sleep problems by decreasing melatonin release. Second, there is a deterioration in social interactions. A lack of regular social interaction can increase stress, depression, and loneliness, and negatively affect sleep quality $[24,25]$. Sleep changes according to confinement vary according to individual personality characteristics. During confinement situations, those with high social participation scores and those with a sense of belonging maintained good sleep quality, whereas those 
who felt lonely or who were older had worse sleep problems [26]. Sleep problems are also associated with all family members who remain at home for long periods of time due to home confinement. It disrupts the boundaries of family members' private lives, interrupts their daily routines and working schedules, and disturbs their circadian rhythms [5]. As the time to go to work and school disappears, bedtime and sleep onset are delayed, sleep duration is reduced, and daytime napping is increased. Frequent and late naps also lower sleep pressure, leading to a delayed bedtime and increased sleep onset latency. Delayed sleep onset also results in a decrease in slow wave sleep, which is affected by circadian factors [27]. Screen time increases during home confinement. A longer screen time is related to shorter sleep duration and lower sleep efficiency [28].

\section{SOCIAL DISTANCING, HOME CONFINEMENT AND MENTAL HEALTH}

Mental health problems can exacerbate sleep problems [29]. The COVID-19 pandemic has resulted in a psychiatric burden to public health. More than half of the general population was emotionally affected by COVID-19, and $16.5 \%$ to $33.7 \%$ experienced depression and anxiety. This related not only to the outbreak itself, but also to preventive measures such as social distancing and home confinement [30-32].

Younger individuals and women were more vulnerable to depression and anxiety [33]. The proportion of UK housewives with mental health problems increased from 23\% in 2017-2019 to 37\% in late April 2020. This was one month after the COVID-19 pandemic [34]. After the COVID-19 outbreak, approximately 7\% of residents of Wuhan, China developed post-traumatic stress disorder (PTSD) symptoms, especially women [35]. Women under the age of 35 and those who received news regarding COVID-19 for at least three hours each day had higher levels of anxiety [35]. People with a history of psychiatric illness may also experience symptoms of anxiety, anger, fear of infection, loneliness, depression, and PTSD more frequently after quarantine [31,36]. During lockdown, anxious people consume more alcohol and have more insomnia [37]. On the other hand, those who arise early in the morning and those who have a good quality of sleep have fewer PTSD symptoms associated with the COVID-19 outbreak [38].

The overall quality of life (QoL) of the general population has declined after the COVID-19 pandemic [39,40]. The decrease in QoL was observed in those who lived in an area where there was an abundance of death, in those who had no contact with others, in those who had a reduced household income, and in those who had reduced PA. It did not deteriorate for those who experienced better sleep time, better quality of sleep, those who were able to spend enough time at home, those who were able to spend more time with their family, and those who had less educational burden [15]. A shift in sleep to the evening was associated with a poor sleep quality and poor QoL. Resilience was positively correlated with age, spiritual beliefs, and family support [17].
One of the most serious mental health problems associated with COVID-19 is suicide [16]. Suicidal thoughts related to the COVID-19 pandemic are linked to both fear of the viral infection itself as well as sleep problems [16]. Suicidal thinking regarding COVID-19 worries is related to the effects of sleep. Long-term isolation, stress, fear, and economic uncertainty leads to sleep and mental health problems. This increases the likelihood of suicidal outcomes. Sleep abnormalities alone may be a risk factor for suicidal ideation, suicide attempts, and suicide deaths. Suicidal thoughts and intentions should be investigated when evaluating individuals with sleep problems [29].

In summary, as there is a bidirectional relationship between sleep and psychiatric comorbidities. Sleep specialists should consider psychiatric comorbidities when dealing with sleep problems [41]. Patients with sleep problems related to home confinement should be screened for mental health problems such as depression, anxiety, and suicidal ideation. Conversely, patients with mental health problems require screening for sleep disturbances [42].

\section{MANAGEMENT OF SLEEP DURING SOCIAL DISTANCING AND HOME CONFINEMENT DUE TO THE COVID-19 PANDEMIC}

In this section, methods to solve sleep problems caused by long-term social distancing and home confinement, which are preventive measures against COVID-19 would be offered. First, the active pursuit of social relationships helps solve sleep problems. Social interaction and social support can relieve stress and improve sleep quality $[43,44]$. Reducing the feeling of loneliness for people who lived alone prior to the COVID-19 outbreak is beneficial in improving both sleep problems and mental health. Social support for people quarantined in their homes for 14 days after contact with a COVID-19 patient lowered their anxiety and stress levels and improved their sleep quality $[26,45]$. Second, positive thinking and the effective use of time provided to an individual as a result of home confinement can be beneficial. Even if less time is spent at work or school, improvements in sleep quality can be produced by engaging in additional outside activities during the day or by participating in more exercise than usual. During social distancing, meetings and gatherings for dining that are not immediately necessary can be reduced. This can reduce work stress, increase work efficiency, and improve nighttime sleep [46]. During home confinement, time is relatively flexible to attend school and work. Therefore, sleep and work schedules can be more closely aligned to an individual's own endogenous dayand night rhythm $[47,48]$ PA can act as an environmental timekeeper and increase psychological resilience against COVID-19 $[49,50]$. It also significantly reduces anxiety and depression and improves sleep quality [23,50-52].

The following methods combining the recommendations of task a task force of the European CBT-I Academy and The Society of Behavioral Sleep Medicine and they would be helpful to 
prevent and treat sleep problems caused by social distancing and home confinement during the COVID-19 pandemic [23,53].

1) Maintain regular sleep and wake-up times.

2) Set a brief time of the day (15 minutes) for reflection.

3) Use the bed only for sleeping and stay out of the bedroom when not sleeping

4) Follow natural sleep rhythms and circadian preferences unless it is a major problem in daily life.

5) Use social media appropriately to share positive information and reduce the stress of daily life.

6) Find a helpful distraction and immerse yourself in it.

7) Exercise outdoors regularly. If possible, walk for at least 30-60 minutes in the morning in natural daylight. This is beneficial.

8) Before bedtime, do something familiar and relaxing.

9) Avoid eating within 2 hours of bedtime.

10) Avoid alcohol.

11) For older people, communicate frequently with family/ friends and avoid napping, if possible.

12) Do not use a mobile phone or other electronic screen near bedtime.

Treatment should be considered if sleep problems persist despite following the above measures. Many sleep specialists recommend cognitive behavioral therapy for insomnia (CBT-I) as the first choice for insomnia. If CBT-I is ineffective or if the effect is weak, short-term medication may be considered. Use of a hypnotic benzodiazepine receptor agonist or benzodiazepine may be considered. If comorbid psychiatric disorders are present, a sedating antidepressant may be used.

\section{CONCLUSION}

The COVID-19 pandemic has brought about many changes in our lives. Preventive measures such as social distancing and home confinement to control the COVID-19 outbreak have resulted in changes to our daily lives and economic conditions. This has resulted in psychological distress, mental health difficulties, and sleep problems. Sleep problems are common in the general population during social distancing and home confinement. They have a bidirectional relationship with anxiety, depression, and suicide. When physicians evaluate patients, it is necessary to screen for sleep problems. When evaluating patients with sleep problems, including insomnia, screening for mental health issues such as suicidal ideation, anxiety, and depression is necessary. For the treatment of sleep problems, CBI-I should be considered as a first-line treatment. Medication can be considered for a short period of time. Living in the COVID-19 pandemic era, we must maintain a regular sleep-wake schedule, sustain good sleep hygiene, and preserve healthy social interactions and PA. These methods will assist in retaining healthy sleep patterns and mental health.

Acknowledgments

None
Conflicts of Interest

The author has no potential conflicts of interest to disclose.

ORCID iD

Wan Seok Seo (D)

https://orcid.org/0000-0002-5122-5360

\section{REFERENCES}

1. Zhu N, Zhang D, Wang W, Li X, Yang B, Song J, et al. A novel coronavirus from patients with pneumonia in China, 2019. New Engl J Med 2020;382: 727-733.

2. Triggle CR, Bansal D, Farag EABA, Ding H, Sultan AA. COVID-19: learning from lessons to guide treatment and prevention interventions. mSphere 2020;5:e00317-e00320.

3. Morin CM, Carrier J, Bastien C, Godbout R; Canadian Sleep and Circadian Network. Sleep and circadian rhythm in response to the COVID-19 pandemic. Can J Public Health 2020;111:654-657.

4. Sinha M, Pande B, Sinha R. Impact of COVID-19 lockdown on sleep-wake schedule and associated lifestyle related behavior: a national survey. J Public Health Res 2020;9:1826.

5. Altena E, Baglioni C, Espie CA, Ellis J, Gavriloff D, Holzinger B, et al. Dealing with sleep problems during home confinement due to the COVID-19 outbreak: practical recommendations from a task force of the European CBT-I Academy. J Sleep Res 2020;29:e13052.

6. Galli F, Reglero G, Bartolini D, Visioli F. Better prepare for the next one. Lifestyle lessons from the COVID-19 pandemic. Pharmanutrition 2020; 12:100193

7. Medic G, Wille M, Hemels MEH. Short- and long-term health consequences of sleep disruption. Nat Sci Sleep 2017;9:151-161.

8. Pfefferbaum B, North CS. Mental health and the Covid-19 pandemic. New Engl J Med 2020;383:510-512.

9. Park KH, Kim AR, Yang MA, Lim SJ, Park JH. Impact of the COVID-19 pandemic on the lifestyle, mental health, and quality of life of adults in South Korea. Plos One 2021;16:e0247970.

10. Mintzer J, Donovan KA, Kindy AZ, Lock SL, Chura LR, Barracca N. Lifestyle choices and brain health. Front Med (Lausanne) 2019;6:204.

11. Razzoli M, Pearson C, Crow S, Bartolomucci A. Stress, overeating, and obesity: insights from human studies and preclinical models. Neurosci Biobehav Rev 2017;76:154-162.

12. Arora T, Grey I. Health behaviour changes during COVID-19 and the potential consequences: a mini-review. J Health Psychol 2020;25:1155-1163.

13. Capers PL, Fobian AD, Kaiser KA, Borah R, Allison DB. A systematic review and meta-analysis of randomized controlled trials of the impact of sleep duration on adiposity and components of energy balance. Obes Rev 2015;16:771-782.

14. Kalmbach DA, Cuamatzi-Castelan AS, Tonnu CV, Tran KM, Anderson JR, Roth T, et al. Hyperarousal and sleep reactivity in insomnia: current insights. Nat Sci Sleep 2018;10:193-201.

15. Cellini N, Canale N, Mioni G, Costa S. Changes in sleep pattern, sense of time and digital media use during COVID-19 lockdown in Italy. J Sleep Res 2020;29:e13074.

16. Killgore WDS, Cloonan SA, Taylor EC, Fernandez F, Grandner MA, Dailey NS. Suicidal ideation during the COVID-19 pandemic: the role of insomnia. Psychiatry Res 2020;290:113134.

17. Voitsidis P, Gliatas I, Bairachtari V, Papadopoulou K, Papageorgiou G, Parlapani E, et al. Insomnia during the COVID-19 pandemic in a Greek population. Psychiatry Res 2020;289:113076.

18. Jahrami H, BaHammam AS, Bragazzi NL, Saif Z, Faris M, Vitiello MV. Sleep problems during the COVID-19 pandemic by population: a systematic review and meta-analysis. J Clin Sleep Med 2021;17:299-313.

19. Jackson CL, Johnson DA. Sleep disparities in the era of the COVID-19 pandemic highlight the urgent need to address social determinants of health like the virus of racism. J Clin Sleep Med 2020;16:1401-1402.

20. Palagini L, Gemignani A, Banti S, Manconi M, Mauri M, Riemann D. Chronic sleep loss during pregnancy as a determinant of stress: impact on pregnancy outcome. Sleep Med 2014;15:853-859. 
21. Sivertsen B, Hysing M, Dørheim SK, Eberhard-Gran M. Trajectories of maternal sleep problems before and after childbirth: a longitudinal populationbased study. BMC Pregnancy Childbirth 2015;15:129.

22. Fuller PM, Gooley JJ, Saper CB. Neurobiology of the sleep-wake cycle: sleep architecture, circadian regulation, and regulatory feedback. J Biol Rhythms 2006;21:482-493.

23. Ernstsen L, Havnen A. Mental health and sleep disturbances in physically active adults during the COVID-19 lockdown in Norway: does change in physical activity level matter? Sleep Med 2021;77:309-312.

24. McHugh JE, Lawlor BA. Perceived stress mediates the relationship between emotional loneliness and sleep quality over time in older adults. Brit J Health Psychol 2013;18:546-555.

25. Wakefield JRH, Bowe M, Kellezi B, Butcher A, Groeger JA. Longitudinal associations between family identification, loneliness, depression, and sleep quality. Br J Health Psychol 2020;25:1-16.

26. Xiao H, Zhang Y, Kong DS, Li SY, Yang NX. Social capital and sleep quality in individuals who self-isolated for 14 days during the coronavirus disease 2019 (COVID-19) outbreak in January 2020 in China. Med Sci Monit 2020; 26:e923921.

27. Borbély AA, Daan S, Wirz-Justice A, Deboer T. The two-process model of sleep regulation: a reappraisal. J Sleep Res 2016;25:131-143.

28. Christensen MA, Bettencourt L, Kaye L, Moturu ST, Nguyen KT, Olgin JE, et al. Direct measurements of smartphone screen-time: relationships with demographics and sleep. PLoS One 2016;11:e0165331.

29. Sher L. COVID-19, anxiety, sleep disturbances and suicide. Sleep Med 2020;70:124.

30. Wang CY, Pan RY, Wan X, Tan Y, Xu L, Ho CS, et al. Immediate psychological responses and associated factors during the initial stage of the 2019 coronavirus disease (COVID-19) epidemic among the general population in China. Int J Environ Res Public Health 2020;17:1729.

31. Salari N, Hosseinian-Far A, Jalali R, Vaisi-Raygani A, Rasoulpoor S, Mohammadi M, et al. Prevalence of stress, anxiety, depression among the general population during the COVID-19 pandemic: a systematic review and meta-analysis. Global Health 2020;16:57.

32. Jahrami H, BaHammam AS, AlGahtani H, Ebrahim A, Faris M, AlEid K, et al. The examination of sleep quality for frontline healthcare workers during the outbreak of COVID-19. Sleep Breath 2021;25:503-511.

33. Taylor MR, Agho KE, Stevens GJ, Raphael B. Factors influencing psychological distress during a disease epidemic: data from Australia’s first outbreak of equine influenza. BMC Public Health 2008;8:347.

34. Daly M, Sutin AR, Robinson E. Longitudinal changes in mental health and the COVID-19 pandemic: evidence from the UK Household Longitudinal Study. Psychol Med 2020:1-10.

35. Huang Y, Zhao N. Generalized anxiety disorder, depressive symptoms and sleep quality during COVID-19 outbreak in China: a web-based cross-sectional survey. Psychiatry Res 2020;288:112954.

36. Jeong H, Yim HW, Song YJ, Ki M, Min JA, Cho J, et al. Mental health status of people isolated due to Middle East respiratory syndrome. Epidemiol Health 2016;38:e2016048.

37. Stein MD, Friedmann PD. Disturbed sleep and its relationship to alcohol use. Subst Abus 2005;26:1-13.

38. Liu N, Zhang F, Wei C, Jia Y, Shang Z, Sun L, et al. Prevalence and predictors of PTSS during COVID-19 outbreak in China hardest-hit areas: gender differences matter. Psychiatry Res 2020;287:112921.

39. Azizi A, Achak D, Aboudi K, Saad E, Nejjari C, Nouira Y, et al. Health-related quality of life and behavior-related lifestyle changes due to the COVID-19 home confinement: dataset from a Moroccan sample. Data Brief 2020;32: 106239.

40. Riiser K, Helseth S, Haraldstad K, Torbjørnsen A, Richardsen KR. Adolescents' health literacy, health protective measures, and health-related quality of life during the Covid-19 pandemic. PLoS One 2020;15:e238161.

41. Janati Idrissi A, Lamkaddem A, Benouajjit A, Ben El Bouaazzaoui M, El Houari F, Alami M, et al. Sleep quality and mental health in the context of COVID-19 pandemic and lockdown in Morocco. Sleep Med 2020;74:248253.

42. Casagrande M, Favieri F, Tambelli R, Forte G. The enemy who sealed the world: effects quarantine due to the COVID-19 on sleep quality, anxiety, and psychological distress in the Italian population. Sleep Med 2020;75:1220.

43. Kent de Grey RG, Uchino BN, Trettevik R, Cronan S, Hogan JN. Social support and sleep: a meta-analysis. Health Psychol 2018;37:787-798.

44. DeVries AC, Glasper ER, Detillion CE. Social modulation of stress responses. Physiol Behav 2003;79:399-407.

45. Uvais NA Moideen S, Hafi B, Rajagopal S, V Maheshwari, Gafoor TA. Insomnia among active patients with COVID-19: a cross-sectional study. Chronobiol Med 2021;3:31-34.

46. Akerstedt T, Knutsson A, Westerholm P, Theorell T, Alfredsson L, Kecklund G. Sleep disturbances, work stress and work hours: a cross-sectional study. J Psychosom Res 2002;53:741-748.

47. Basner M, Fomberstein KM, Razavi FM, Banks S, William JH, Rosa RR, et al. American time use survey: sleep time and its relationship to waking activities. Sleep 2007;30:1085-1095.

48. Wheaton AG, Chapman DP, Croft JB. School start times, sleep, behavioral, health, and academic outcomes: a review of the literature. J Sch Health 2016; 86:363-381.

49. Killgore WDS, Taylor EC, Cloonan SA, Dailey NS. Psychological resilience during the COVID-19 lockdown. Psychiat Res 2020;291:113216.

50. Holmes PV. Trophic mechanisms for exercise-induced stress resilience: potential role of interactions between BDNF and galanin. Front Psychiatry 2014;5:90.

51. Schuch FB, Stubbs B, Meyer J, Heissel A, Zech P, Vancampfort D, et al. Physical activity protects from incident anxiety: a meta-analysis of prospective cohort studies. Depress Anxiety 2019;36:846-858.

52. Kredlow MA, Capozzoli MC, Hearon BA, Calkins AW, Otto MW. The effects of physical activity on sleep: a meta-analytic review. J Behav Med 2015; 38:427-449.

53. Crew EC, Baron KG, Grandner MA, Ievers-Landis CE, McCrae CS, Nadorff MR, et al. The Society of Behavioral Sleep Medicine (SBSM) COVID-19 task force: objectives and summary recommendations for managing sleep during a pandemic. Behav Sleep Med 2020;18:570-572. 